PAPER

\section{A sensitive detection method for high resolution spectroscopy of trapped antihydrogen, hydrogen and other trapped species}

To cite this article: Claudio Lenz Cesar 2016 J. Phys. B: At. Mol. Opt. Phys. 49074001

View the article online for updates and enhancements.
Related content

- Aspects of 1S-2S spectroscopy of trapped
$\frac{\text { antihydrogen atoms }}{\text { C Ø Rasmussen, N Madsen and F }}$
Robicheaux
- Physics with antihydrogen
W A Bertsche, E Butler, M Charlton et al.
- Ultra-low energy antihydrogen
M H Holzscheiter and M Charlton

\section{Recent citations}

\author{
Special issue on antihydrogen and \\ positronium \\ Mike Charlton et al
}

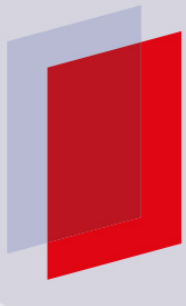

\section{IOP ebooks'}

Bringing you innovative digital publishing with leading voices to create your essential collection of books in STEM research. Start exploring the collection - download the first chapter of every title for free. 


\title{
A sensitive detection method for high resolution spectroscopy of trapped antihydrogen, hydrogen and other trapped species
}

\author{
Claudio Lenz Cesar \\ Instituto de Física, Universidade Federal do Rio de Janeiro, Caixa Postal 68528, 21941-972 Rio de Janeiro, \\ RJ, Brazil \\ E-mail: lenz@if.ufrj.br
}

Received 14 September 2015, revised 8 December 2015

Accepted for publication 9 December 2015

Published 11 March 2016

\begin{abstract}
A method for detection of the weak $1 \mathrm{~s}-2 \mathrm{~s}$ laser excitation of a few trapped antihydrogen atoms is described. It involves the typical antihydrogen trapping environment that combines a magnetic trap for the atoms as well as a Penning trap for its constituent particles. By photoionization of the excited state the photoion can be kept in a weak Penning trap and at a suitable time be ejected towards a charged particle detector such as a microchannel plate or a channel electron multiplier. Since it does not rely on annihilation, the method is also suitable for trapped hydrogen and may find application with other species when a weak transition to a metastable state is intended and only a few trapped atoms or molecules are available.
\end{abstract}

Keywords: antihydrogen, high resolution laser spectroscopy, atom trap, Penning trap

(Some figures may appear in colour only in the online journal)

\section{Introduction}

Cold antihydrogen has been produced [1-3] and trapped at low numbers [4-6]. The main goal of the ALPHA and ATRAP experiments at CERN is a high precision test of the charge-parity-time (CPT) symmetry by comparing the $1 \mathrm{~s}-2 \mathrm{~s}$ spectra of the antiatom and its conjugate atom. While the aimed precision of this spectroscopic comparison is parts in $10^{15}$ as done with hydrogen [7], and beyond, the initial challenge is the detection of the optical transition. In the ALPHA experiment-taken as the platform for this articlethe magnetic trap confines the antihydrogen atoms to a diameter of $44 \mathrm{~mm}$, while the laser beam waist could be set in the range $100-300 \mu \mathrm{m}$, depending on the enhancement cavity length and configuration. Given the ratio of these diameters one can expect a low frequency at which the atom will cross the laser beam. A Monte Carlo simulation can estimate the time between crosses to around tens of milliseconds [8]. These long times together with a low transition rate lead to an integration time in the range of tens to hundreds of seconds in order to achieve unity excitation probability summed over the many passages. With the current state of the art production rate, only a few antiatoms $(\sim 0-4)$ are produced in each trapping cycle lasting minutes. Limited by time-of-flight broadening and the residual Zeeman effect, the first spectrawithout any extra cooling of the sample initially trapped at the $540 \mathrm{mK}$ deep trap-should have a fractional resolution of $10^{-11}$ [8]. The apparatus is essentially ready for attempting the first laser spectroscopy of an antiatom, a major breakthrough, but the detection of the transition remains a big challenge within the timescale of a typical yearly run at CERN's Antiproton Decelerator.

Atomic hydrogen spectroscopy in the $1 \mathrm{~s}-2 \mathrm{~s}$ transition has been performed both in a trap [9] and in cryogenic beams which now reach an accuracy of parts in $10^{15}$ [7]. Once excited to the $2 \mathrm{~s}$ state, this metastable state can be quenched by applying an electric field that mixes it with the $2 p$ state resulting in a fast Lyman- $\alpha$ fluorescence photon. Typically, the experiments with hydrogen detect this Lyman- $\alpha$ photon, usually with a large number of atoms in the laser beam and 

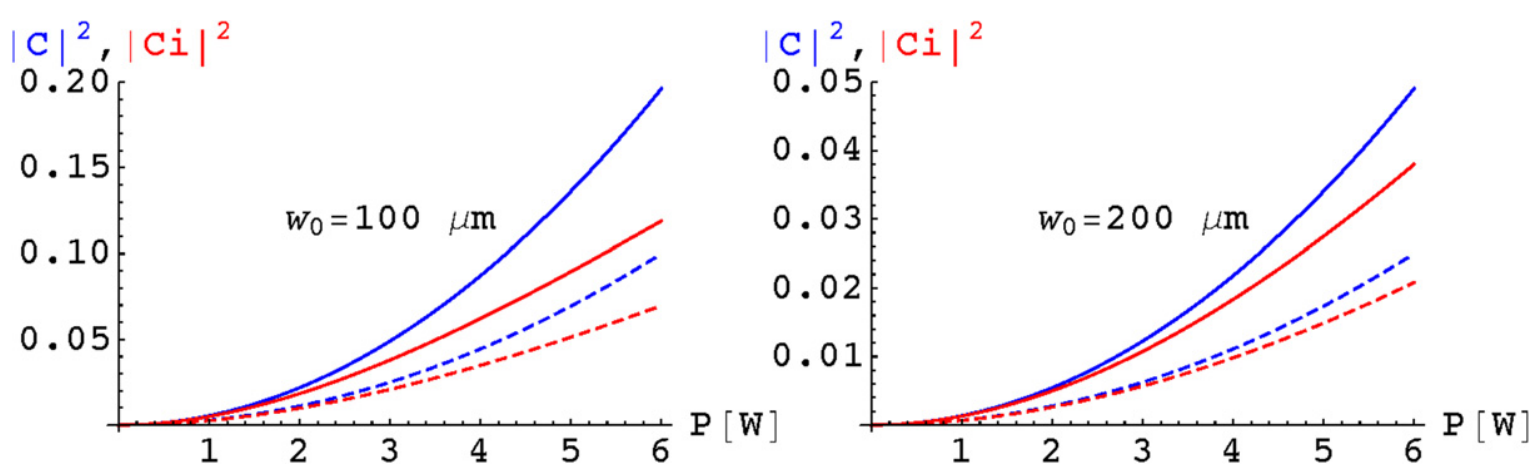

Figure 1. Excitation probability for a single passage through the laser beam axis $\left(\rho_{L}=0\right)$ as a function of the laser power using equations (7) (in blue) and 9 (in red), at resonance $\left(\delta \omega_{c a}=0\right)$ and with transverse speeds $v_{L}=50 \mathrm{~ms}^{-1}$ (solid lines) and $v_{L}=70 \mathrm{~ms}^{-1}$ (dashed lines), for beam waists $w_{0}=100 \mu \mathrm{m}$ (left plot) and $w_{0}=200 \mu \mathrm{m}$ (right plot). The difference $|C|^{2}-\left|C_{i}\right|^{2}$ gives the ionization probability. See appendix for definitions and level of validity of the approximations used.

with enough solid angle for collection of the Lyman- $\alpha$ photon. In the case of antihydrogen the setups are already complex around the trap region to incorporate a Lyman- $\alpha$ detector and the number of atoms is of order unity.

With the annihilation detector in ALPHA, one of the possible detection schemes is to look for annihilation once the excited atom decays to a non-trapped state, but calculations have shown a low probability for this process at the high magnetic fields present in the trap [10]. Another detection possibility comes from the photoionization that takes place together with the excitation: a single $243 \mathrm{~nm}$ photon has sufficient energy to ionize the $2 \mathrm{p}$ state. Typically, in the cycle at ALPHA, after the atoms have been made, the Penning trap is dropped and all the charged particles, positrons and antiprotons and leftover electrons are dumped. With the Penning trap off, the antiproton resulting from the photoionization process would leave the trap area and annihilate. This annihilation signal, in the so called 'appearance mode', could be looked for. Even if an extra detector would be installed around the annihilation region, a difficulty arises from the detector dark count, since this signal could come at any time while the laser is interacting with the atoms, i.e., minutes. In the other 'disappearance mode' one looks for losses of trapped atoms at the end of the cycle with the quick dump of the magnetic trap. Both these modes, albeit with a neutral atom annihilation, were demonstrated with a microwave spin-flip transition at ALPHA [11]. In this paper I suggest another scheme $[12,13]$, which saves the photoionized antiprotons in a weak Penning trap restored just before the laser is introduced in the trap. The photoionized antiprotons stored for the whole cycle of the laser interaction could then be dumped either at a microchannel plate (MCP) detector or at a channel electron multiplier (CEM) in a defined fast time window. This would result in a very small dark count from the detectors. Using an MCP or a CEM, which do not rely on annihilation, the method can be extended to atoms and molecules, hydrogen being a prime candidate. In the next section I discuss photoionization rates, the photoion leftover energy and the required Penning traps, and broadening mechanisms. I consider the case for atoms and molecules in section 3 and superficially describe a way to trap hydrogen in the same apparatus that traps antihydrogen.

\section{Method, rates and values}

In this section I discuss in more detail the method for trapping the photoionized antiproton, the antihydrogen photoionization rates and values for the Penning and the magnetic trap. As mentioned before, the antihydrogen trapping is made in an environment superposing a magnetic trap for the neutral atoms as well as a Penning trap for the charged particles necessary for the production of cold antihydrogen: positrons, antiprotons, and electrons which are used for initial cooling of the antiprotons. The ALPHA experiment has typically proceeded with the following steps: antiprotons are trapped, electron cooled, manipulated and stored in one well; positrons are trapped in a neighboring well after many manipulations; electrons are expelled out before mixing the antiprotons with the positrons; a mixing phase gently carries the antiprotons into the positron cloud resulting in the formation of antihydrogen; and the Penning trap is then turned off leaving only possibly trapped neutral atoms. At this point, the laser interaction can begin.

The $1 \mathrm{~s}-2 \mathrm{~s}$ transition is excited via two counterpropagating photons at $243 \mathrm{~nm}$, so as to achieve Doppler-free resolution. Since the transition is weak and requires counterpropagating beams, an optical enhancement cavity is typically employed. Nowadays a commercial laser (TOPTICA Photonics AG) can deliver $100 \mathrm{~mW}$ of $\mathrm{cw}$ power at $243 \mathrm{~nm}$. With a well designed optical cavity, an enhancement of $20-50$ can be envisioned so that one can foresee power levels of up to $5 \mathrm{~W}$. While the $1 \mathrm{~s}-2 \mathrm{~s}$ excitation proceeds, with the atom within the laser beam, the 2 s ionization by a single $243 \mathrm{~nm}$ photon can occur. In the appendix the calculation of these rates is developed analytically, in the perturbative regime, for a single passage through the laser beam. Despite being in the perturbative regime, and not always valid, analytical expressions are useful and help in getting a better understanding of the processes. Typical results are shown in figure 1 for the probability of finding the atom in the excited 


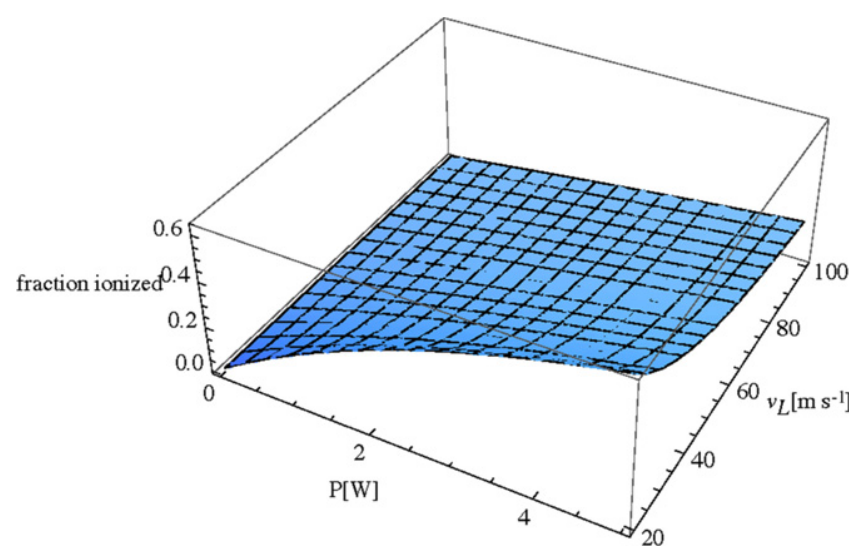

Figure 2. Fraction of ionized atoms to excited ones as a function of laser power and beam crossing speed for a beam waist of $100 \mu \mathrm{m}$ and supposing that the atom goes through the center of the laser beam as estimated from equations (7) and (9) with $\rho_{L}=0$. See appendix for details.

state after a passage through the laser beam, without accounting for ionization $\left(|C|^{2}\right)$ and with ionization $\left(\left|C_{i}\right|^{2}\right)$.

With a beam waist of $100 \mu \mathrm{m}$ and a power of $5 \mathrm{~W}$, over $34 \%$ of the excited atoms with a speed of $50 \mathrm{~m} \mathrm{~s}^{-1}$ transverse to the laser beam are photoionized. Faster atoms are ionized at lower fractions. For the highest speed possible in the ALPHA trap, $v_{L}=94 \mathrm{~m} \mathrm{~s}^{-1}$ corresponding to a kinetic energy of $540 \mathrm{mK}$, the ionized fraction of the excited atoms is about $20 \%$. A graph of the ionization fraction is shown in figure 2 . It can be seen that the ionized fraction increases for low speeds. The curve should not be considered quantitatively at low speeds because equations (7) and (9) lose accuracy, as explained in the appendix.

In cases where the atom is excited but not ionized, the atom may go back to the ground state and be subjected to other cycles if it remains trapped; or it may be ejected from the trap in the case of a spin-flip. On average, at the end of a long cycle, ionization would be the best chance of detecting an excitation. In the velocity range considered, the ionization fraction is still small for low laser powers (see figure 2) and this detection process would benefit from higher laser intensities.

Once the milestone of an optical signal has been established and the experiment proceeds towards very high precision, both a high ionization rate and a high excitation rate have to be avoided as they will broaden the transition if they are higher than the other dominating experimental linewidths, such as time-of-flight, residual Zeeman, ac Stark, or the natural decay rate of $8 \mathrm{~s}^{-1}$. But, by then, one hopes to be producing higher rates of trapped atoms and to be cooling the atoms-with Lyman- $\alpha$ or adiabatic cooling, for exampleand lowering the trap bias magnetic field where other detection mechanisms, such as spin-flip, are more efficient. Lineshapes in the ultracold atomic regime at low magnetic fields have been worked out for a particular trap in [13, 14] and are not the focus here.

The $5.1 \mathrm{eV}$ photon of $243 \mathrm{~nm}$ will ionize the $2 \mathrm{~s}$ state with an excess energy of $1.7 \mathrm{eV}$. Since the positron is so much lighter than the nucleus, conservation of momentum and energy dictates that it will take most of the energy, leaving only a fraction of $\sim 1 / 1837$ of that for the antiproton, or about $1 \mathrm{meV}$. That means that even a very weak Penning trap could keep these antiprotons. The typical cyclotron orbit for such an antiproton would be micrometric at ALPHA's bias field of $1 \mathrm{~T}$. Even if this field is lowered to tens of gauss, that should not present much of a problem for the trapping, provided that the other trapping fields (octupole, in the case of ALPHA) are also lowered.

The electric field to reestablish the Penning trap for the purpose of trapping the photoionized antiproton presents a negligible effect on the $2 \mathrm{~s}$ state metastability during a single passage through the laser. In the zero magnetic field, the $2 \mathrm{~s}$ decay rate is increased by electric fields as $\gamma_{2 s} \approx \gamma_{2 p}\left(E\left[V \mathrm{~cm}^{-1}\right] / 475\right)^{2}$ due to the near degeneracy with the $2 \mathrm{p}_{1 / 2}$ state, separated by the Lamb shift [15]. A field of $1 \mathrm{~V} \mathrm{~cm}$ would bring the decay rate to

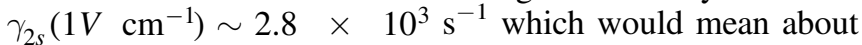
$1 \%$ of decay probability for an excited atom over the crossing time of a $50 \mathrm{~m} \mathrm{~s}^{-1}$ atom through the laser beam with a $w_{0}=100 \mu \mathrm{m}$ beam waist. Thus, fields of this order pose no problem for detectability, though it should be considered in the calculation for the lineshape if one will be splitting the line for an absolute frequency measurement. The relativistic field seen by the atoms in the large bias magnetic field presently at ALPHA is also of this order $E_{\text {rel }}=$ $|\vec{v} \times \vec{B}|=v_{L} B_{\text {bias }}$, for $v_{L}=50 \mathrm{~m} \mathrm{~s}^{-1}$ in a $1 \mathrm{~T}$ field, $E_{\text {rel }}=0.5 \mathrm{~V} \mathrm{~cm}^{-1}$. On the other hand, at such high bias field the quenched decay rate of the $2 \mathrm{~s}$ state gets drastically reduced from the zero magnetic field value by the large Zeeman separation from the various $2 p$ states [16].

Specific situations, depending on the local magnetic and electric field where the atom crosses the laser beam, can be incorporated into a Monte Carlo simulation. Such a comprehensive numerical simulation with these rates, taking into account the magnetic field in the ALPHA experimental configuration, and including the non-perturbative regime, have been done by ALPHA members [10]. The goal here is just to present the idea and viability of this technique.

Finally, it is necessary to consider the detection of the antiproton. The ALPHA annihilation detector can detect and image a single antiproton annihilation at the trap walls with about $70 \%$ efficiency around the trap center. But, being ionized on the axis of the main solenoid, releasing this cold antiproton radially towards the wall is not a simple task. A simpler detection would involve the use of the MCP already employed in the experiment to detect positrons, electrons and antiprotons and measure their energy distribution, or temperature. The particles are dumped along the magnetic field axis. A single antiproton is detectable by the MCP. A question not completely resolved experimentally, though, is the discrimination of a few antiprotons in a single dump. The antiprotons would be coming along the same magnetic field axis (the laser is almost aligned with the main solenoid) and the release of annihilation energy in the form of charged pions generate tracks, causing a blurring of the MCP image. This may lead to difficulties in distinguishing 1, 2 or 3 antiprotons. 
Solving this issue may be just a question of combining image analysis and integrated contrast. A slow dump may also solve this issue separating the particles in time, either with an MCP or with a CEM. In any case, with the numbers above, this should not be a problem for the initial detection of the laser excitation as one typically has less than one atom at a time, on average. This issue may be simpler to deal with in the spectroscopy of atoms and molecules in low magnetic fields, which is the topic of the next section.

\section{Atoms and molecules}

In this section I briefly discuss the prospects of using this technique with atoms and molecules. It has been brought to my attention that a proposal along these lines has previously been made in the context of $\mathrm{He}^{+}$spectroscopy in an RF trap where the $\mathrm{He}^{2+}$ would be kept for detection [17], in a situation similar to the one presented here with a very low number of excited ions and long integration times.

The discussion of antihydrogen in the previous sections is also valid for hydrogen. Large hydrogen traps, such as the ones in $[18,19]$, have been discontinued due to their complexities, and none of them integrated a Penning trap. In those traps, the number of hydrogen atoms was large, which permitted laser spectroscopy in the 1s-2s transition [20], and Lyman- $\alpha$ spectroscopy and cooling [19], with the detection of Lyman- $\alpha$ photons, despite the low solid angle for the detector in an apparatus that was optimized for Bose-Einstein condensation rather than for laser spectroscopy. A trial to achieve a large hydrogen trap with the buffer gas technique [21] eventually failed due to the evaporation force of the 'wind' of retreating the buffer gas. But, certainly, a small quantity could have been trapped. Another group [22, 23] also trapped hydrogen and deuterium, at low densities, which were detected via ionization. These trapping schemes could be made to work at interesting levels for high precision spectroscopy using the detection technique here described.

In our group in Brazil we have developed another technique to generate a cryogenic beam suitable to load a magnetic trap of hydrogen and other species, which we call matrix isolation sublimation (MISu) [24-29]. It involves three steps: (i) a solid film of inert gas, such as $\mathrm{Ne}$ or $\mathrm{H}_{2}$, is grown onto a cold sapphire substrate at $\sim 4 \mathrm{~K}$; (ii) atoms are implanted, typically via laser ablation of some suitable solid precursor, such as $\mathrm{LiH}$ for the case of interest in $\mathrm{Li}$ and $\mathrm{H}$, thus forming the matrix isolation; and (iii) the matrix is sublimated at cryogenic temperatures with a heat pulse to the sapphire substrate. Intense beams of atoms and molecules can be generated with this system, at low forward velocity and with a large enough sample at the low energy tail of the distribution such that trapping should be possible with generous numbers $[24,25,30]$, as seen below.

We are setting up two configurations for traps: using permanent magnets with $0.2 \mathrm{~T}$ deep traps, or employing a small switched (or dc) superconducting coil. In the case of a permanent magnet (or a high inductance coil), the trap loading would proceed via collisions in a fast sublimation. The sample leaves the matrix at high density-short mean free path-and as it expands into vacuum, it eventually disconnects, thermally and mechanically, with a long mean free path. If this disconnection happens inside the trap volume, the part of the sample with energy below the trap depth gets trapped. For this process to work, a fully entrained sample of light atoms in the heavier neon matrix atoms is necessary, as the forward speed set by the heavier neon represents little kinetic energy.

Based on this system, we also proposed to trap hydrogen atoms in the ALPHA apparatus, so as to trap hydrogen in the same space where antihydrogen is trapped, allowing for a good control over systematic effects for the direct spectroscopy comparison of hydrogen and antihydrogen. A simplified estimate — based on the model in [24] and on data from [27]—-predicts a sample of more than $10^{8}$ atoms trapped [31] for the magnetic fields in the ALPHA trap. The process is illustrated in figure 3 . The sublimation of the matrix happens at a high value and high gradient of the magnetic field. The sublimation plume travels and expands and, for some configurations, it will typically reach a long mean free path some 2-3 cm away, where it disconnects. Along the way collisions would be promoting a Maxwell-Boltzmann distribution, i.e., generating a low velocity part of the distribution. The detection technique here proposed would work in this case by providing a reference of hydrogen in the same trapping environment, and the number of trapped atoms could be diminished on purpose for checking systematic effects for the laser spectroscopy as well as other experiments with antihydrogen.

For the purpose of other atoms and molecules, the ionizing laser could be a different laser from the spectroscopy laser. This technique would find applications especially where the number of trapped species is very small, with a weak optical transition, and when the integration time for excitation is long. Forbidden transitions to metastable state are typical candidates, such as the $4 d^{10} 5 s^{2} S_{1 / 2}-4 d^{9} 5 s^{2}{ }^{2} D_{5 / 2}$ transition in silver [32], for instance. The MISu technique mentioned above should provide a very general technique to trap species with magnetic moment, including molecules. Joining the magnetic trap with a weak Penning trap, while challenging, can be very useful in the spectroscopy of species at low numbers and excitation rates.

\section{Conclusion and prospects}

A detection technique for the challenging laser spectroscopy in the $1 \mathrm{~s}-2 \mathrm{~s}$ transition on a small number of trapped antihydrogen atoms is proposed. It is based on restoring a weak Penning trap prior to the laser interaction in order to keep the photoionized antiproton, which is later dumped onto a charged particle detector such as a microchannel plate or a channel electron multiplier. The main advantage of the technique is that the detection would happen in a fast well determined time window allowing for a very low background count. The ionization happens automatically as a single $243 \mathrm{~nm}$ photon has enough energy to ionize the $2 \mathrm{~s}$ state. The 

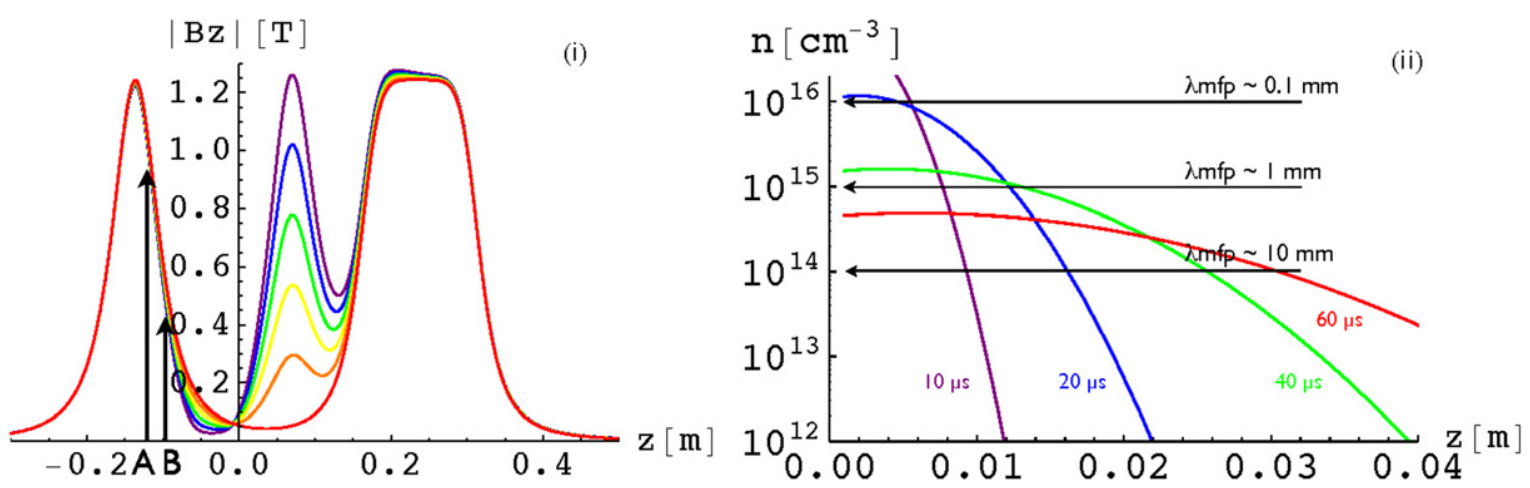

Figure 3. Sketch of the proposed MISu trapping at ALPHA. On the left (i), calculated magnetic field configurations along the axis are shown. The point identified as ' $\mathrm{A}$ ' is where the isolation matrix would be placed. Once the matrix is sublimated the plume will travel to the right and it will disconnect near point ' $\mathrm{B}$ '. The field difference between these points is a measure of the trapping depth. In the right (ii), calculated curves of the density of the matrix gas $(\mathrm{Ne})$ are shown as a function of position for different times, shown in different colors. Notice how at $\mathrm{z}=0.02 \mathrm{~m}$ the density can reach values so that the mean free path $\left(\lambda_{\mathrm{mfp}}\right)$ is about $1 \mathrm{~mm}$ and then quickly decays. A plot (not shown) of the density at a position as a function of time would show a decay time around 50-100 $\mu$ s, quick enough to avoid much Ne evaporation of trapped atoms. See references for more detail.

requirements for the Penning trap are discussed as well as the small quenching effect due to an applied electric field, or the relativistic electric field in the magnetic trap seen by the moving atom. The prospects for using this technique for the initial detection of the first optical transition in an antiatom are promising. We also consider the utilization of this technique for other atoms, particularly hydrogen, and molecules. With the use of the MISu technique, a wide variety of paramagnetic atoms and molecules should be trappable in low amounts. For the case of hydrogen and antihydrogen, the motivation is doing laser spectroscopy of both conjugated species in the same trapping environment. For the case of other atoms and molecules, a technique to trap and perform laser spectroscopy in weak transitions with a small number of species may open new opportunities, making it worth the complexity of integrating a weak Penning trap into a magnetic trap.

\section{Acknowledgments}

I acknowledge discussions with my ALPHA colleagues and colleagues from UFRJ who joined in the FAPERJ grant proposal [13]. This work is partially supported by the Brazilian institutions FAPERJ, CNPq and RENAFAE.

\section{Appendix}

In this appendix the excitation and photoionization rates for (anti)hydrogen are developed. Let us consider the $1 \mathrm{~s}-2 \mathrm{~s}$ excitation, by taking the atom with states $|A\rangle,\left|B^{\prime} s\right\rangle$ and $|C\rangle$, where $|A\rangle$ is the $1 \mathrm{~s} \mathrm{ket},|C\rangle$ is the $2 \mathrm{~s}$ ket, and the $\left|B^{\prime} s\right\rangle$ are the intermediate states for electric dipole allowed transitions: $2 \mathrm{p}$, $3 p$, and so on. One can write the atom's wavefunction in the interaction picture as:

$$
\begin{aligned}
|\Psi(t)\rangle= & A(t)|A\rangle+B(t) \exp \left(-\mathrm{i} \omega_{b a} t\right)|B\rangle \\
& +C(t) \exp \left(-\mathrm{i} \omega_{c a} t\right)|C\rangle
\end{aligned}
$$

Assuming a quasi-collimated Gaussian beam (long divergence length) in a counterpropagating configuration where the two photons will be absorbed from opposite beams the spatial phases from the two fields, given by the wavevector propagation and the field curvature, cancel, and so does the first-order Doppler effect. One can use standard second-order perturbation theory, or take equation 7 of [13] as a starting point. Going to the atom's reference and supposing that it is going unperturbed (in its external motion) through the laser beam at an impact parameter $\rho_{L}$-with closest approach supposed at $\mathrm{t}=0$ - and speed $v_{L}$ transverse to the laser beam, one can write for the amplitude of probability of the excited state starting from time $t_{i}$ in state $|A\rangle$ :

$$
\begin{aligned}
C(t)= & 2 \times \sum_{b=2,3 \ldots} \frac{1}{\mathrm{i} \hbar)^{2}} \int_{t_{i}}^{t} \mathrm{~d} t_{2} \int_{t_{i}}^{t_{2}} \mathrm{~d} t_{1} \frac{\vec{\mu}_{a b} \cdot \vec{\epsilon}}{2} \frac{\vec{\mu}_{b c} \cdot \vec{\epsilon}}{2} \\
& \times E_{0}^{2} \exp \left[-2 \frac{\rho_{L}^{2}}{w_{0}^{2}}\right] \\
& \times \exp \left[-\frac{\left(v_{L} t_{1}\right)^{2}}{w_{0}^{2}}\right] \exp \left[-\frac{\left(v_{L} t_{2}\right)^{2}}{w_{0}^{2}}\right] \\
& \times \exp \left[\mathrm{i}\left(\omega_{b a}-\omega\right) t_{1}\right] \exp \left[\mathrm{i}\left(\omega_{c b}-\omega\right) t_{2}\right],
\end{aligned}
$$

where the first factor of 2 comes from the permutation of the laser beams, $\mu_{b c}$ and $\mu_{a b}$ are the dipole moments for the transitions, $\omega_{c b}$ and $\omega_{b a}$ are the Bohr frequencies between the states, and the laser field parameters [33] $\vec{\epsilon}, E_{0}$ and $w_{0}$ are the laser polarization that we take as linear and the same for both counterpropagating beams, the electric field amplitude, and the laser beam waist, respectively. It is timely to remember 
that the Gaussian dependency of the electric field is given by $\exp \left(-\rho^{2} / w_{0}^{2}\right)$ and so the intensity will depend on $\exp \left(-2 \rho^{2} / w_{0}^{2}\right)$, where $\rho^{2}(t)=\rho_{L}^{2}+\left(v_{L} t\right)^{2}$ is the distance to the laser axis, taken to coincide with the $z$-axis, squared.

Only when $t_{2} \sim t_{1}$ (simultaneous absorption of both photons) in all the slow varying elements is the integral of non-negligible value. Thus, we take the Gaussian part outside the $t_{1}$ integral by substituting $t_{1} \rightarrow t_{2}$ in this term:

$$
\begin{aligned}
C(t)= & \frac{E_{0}^{2}}{2(i \hbar)^{2}} \exp \left[-2 \frac{\rho_{L}^{2}}{w_{0}^{2}}\right] \sum_{b=2,3 \ldots}\left(\vec{\mu}_{a b} \cdot \vec{\epsilon}\right)\left(\vec{\mu}_{b c} \cdot \vec{\epsilon}\right) \\
& \times \int_{t_{i}}^{t} \mathrm{~d} t_{2} \exp \left[\mathrm{i}\left(\omega_{c b}-\omega\right) t_{2}\right] \exp \left[-2 \frac{\left(v_{L} t_{2}\right)^{2}}{w_{0}^{2}}\right] \\
& \times \int_{t_{i}}^{t_{2}} \mathrm{~d} t_{1} \exp \left[\mathrm{i}\left(\omega_{b a}-\omega\right) t_{1}\right] .
\end{aligned}
$$

The integral in $t_{1}$ is trivial and produces: $I_{t_{1}}=$ $\int_{t_{i}}^{t_{2}} \mathrm{~d} t_{1} \exp \left[\mathrm{i}\left(\omega_{b a}-\omega\right) t_{1}\right]=i \quad\left(\exp \left[\mathrm{i}\left(\omega_{b a}-\omega\right) t_{2}\right]-\exp \right.$ $\left.\left[\mathrm{i}\left(\omega_{b a}-\omega\right) t_{i}\right]\right) /\left(\omega-\omega_{b a}\right)$. The second term, in $t_{i}$, is nonresonant once carried into the $t_{2}$ integral and can be neglected. Thus, one obtains

$$
\begin{aligned}
C(t) & =\frac{E_{0}^{2}}{2(\mathrm{i} \hbar)^{2}} \exp \left[-2 \frac{\rho_{L}^{2}}{w_{0}^{2}}\right] \sum_{b=2,3}\left(\vec{\mu}_{a b} \cdot \vec{\epsilon}\right)\left(\vec{\mu}_{b c} \cdot \vec{\epsilon}\right) \\
& \times \int_{t_{i}}^{t} \mathrm{~d} t_{2} \exp \left[-2 \frac{\left(v_{L} t_{2}\right)^{2}}{w_{0}^{2}}\right] \\
& \times \frac{\exp \left[\mathrm{i}\left(\omega_{c b}+\omega_{b a}-2 \omega\right) t_{2}\right]}{\mathrm{i}\left(\omega_{b a}-\omega\right)} .
\end{aligned}
$$

One can identify $\omega_{c b}+\omega_{b a}=\omega_{c a}$. With the Gaussian dependency on $t_{2}$ one can extend the time limits to $\pm \infty$ and obtain the amplitude of probability for a transition in a single passage through the laser beam:

$$
\begin{aligned}
C(\infty)= & \frac{E_{0}^{2}}{2(\mathrm{i} \hbar)^{2}} \sqrt{\frac{\pi}{2}} \frac{w_{0}}{v_{L}} \exp \left[-2 \frac{\rho_{L}^{2}}{w_{0}^{2}}\right] \\
& \exp \left[-\frac{w_{0}^{2}\left(\omega_{c a}-2 \omega\right)^{2}}{8 v_{L}^{2}}\right] \\
& \times \sum_{b=2,3 \ldots} \frac{\left(\vec{\mu}_{a b} \cdot \vec{\epsilon}\right)\left(\vec{\mu}_{b c} \cdot \vec{\epsilon}\right)}{\mathrm{i}\left(\omega_{b a}-\omega\right)} .
\end{aligned}
$$

In [34] (or [35]) the summation over all intermediate (b) states have been performed and their result for a degenerate two-photon excitation $\left(2 \omega=\omega_{2 s 1 s}\right)$ translates into: $\left|\sum_{b=2,3 \ldots} \frac{\left(\vec{\mu}_{a b} \cdot \vec{\epsilon}\right)\left(\vec{\mu}_{b c} \cdot \vec{\epsilon}\right)}{\mathrm{i}\left(\omega_{b a}-\omega\right)}\right|^{2}=\left(\frac{-11.78}{2} \frac{2 a_{0}^{2} e^{2}}{3 R y / \hbar}\right)^{2}$, where $a_{0}$ is the Bohr radius, $e$ is the electron charge, and $R y$ is the Rydberg energy.
One thus gets for the excitation probability after a single pass through the laser beam:

$$
\begin{aligned}
|C(\infty)|^{2}= & \left(\frac{E_{0}^{2}}{2} \sqrt{\frac{\pi}{2}} \frac{w_{0}}{v_{L}}\right)^{2} \exp \left[-4 \frac{\rho_{L}^{2}}{w_{0}^{2}}\right] \\
& \times \exp \left[-2 \frac{w_{0}^{2}\left(\omega_{c a}-2 \omega\right)^{2}}{8 v_{L}^{2}}\right] \\
& \times \frac{1}{\hbar^{2}}\left(\frac{-11.78}{2} \frac{2 a_{0}^{2} e^{2}}{3 R y}\right)^{2} .
\end{aligned}
$$

Identifying the power $(P)$ and electric field in a Gaussian beam $\left(w_{0}^{2} E_{0}^{2}=4 P /\left(\pi \epsilon_{0} c\right)\right)$, defining a detuning $\delta \omega_{c a}=\omega_{c a}$ $-2 \omega$, and substituting the numbers one obtains:

$$
|C(\infty)|^{2}=\frac{1.362 \times 10^{-7} P^{2}}{v_{L}^{2} w_{0}^{2}} \exp \left[-4 \frac{\rho_{L}^{2}}{w_{0}^{2}}\right] \exp \left[-2 \frac{w_{0}^{2} \delta \omega_{c a}^{2}}{8 v_{L}^{2}}\right]
$$

While this equation is useful for many practical calculations, notice that spontaneous emission from the $2 \mathrm{~s}(C)$ state has been neglected. This is typically justified on most occasions since the atoms cross the laser beam in times much shorter than the $2 \mathrm{~s}$ decay time. In the presence of a quenching electric field, the 2 s decay time is shortened and the decay might need to be taken into account as discussed below. Since the derivation used perturbation theory, this equation is also only valid in the low excitation probability limit, which would be a limiting case at resonance for $P=5 \mathrm{~W}$, with an atomic transversal speed of $50 \mathrm{~m} \mathrm{~s}^{-1}$ and a beam waist $w_{0}=$ $100 \mu \mathrm{m}$.

In order to properly account for the photoionization rate one should employ a density matrix formulation, as in equations $(10)(a-c)$ of [35], and the results would be obtained from numerical calculation. For the purpose of obtaining a rough estimate in analytical form and within the limits of perturbation (supposing most of the population remains in the initial state, e.g., $A(t) \approx 1$ ) I introduce a loss rate by including an imaginary Hamiltonian. I modify equation (4) as a differential equation including a photoionization loss rate $\gamma_{i}(t)=2 \pi \beta_{\text {ioni }}[2 I(t)]$, with [35] $\beta_{\text {ioni }}=1.202 \times 10^{-4} \quad\left[\mathrm{~Hz} \quad\left(\mathrm{Wm}^{-2}\right)^{-1}\right] \quad$ and $I(t)=(1 / 2) \epsilon_{0} c E_{0}^{2} \exp \left[-2 \rho_{L}^{2} / w_{0}^{2}\right] \exp \left[-2\left(v_{L} t\right)^{2} / w_{0}^{2}\right]$ being the laser intensity in each direction in our reference frame, considered equal from both directions. The differential equation for the amplitude of the $C$-state, considering ionization, becomes:

$$
\begin{aligned}
\dot{C}_{i}(t)= & \exp \left[-2 \frac{\rho_{L}^{2}+\left(v_{L} t\right)^{2}}{w_{0}^{2}}+\mathrm{i} \delta \omega_{c a} t\right] \\
& \times \sum_{b=2 \ldots} \frac{E_{0}^{2}}{(\mathrm{i} \hbar)^{2}} \frac{\left(\vec{\mu}_{a b} \cdot \vec{\epsilon}\right)\left(\vec{\mu}_{b c} \cdot \vec{\epsilon}\right)}{2 \mathrm{i}\left(\omega_{b a}-\omega\right)} \\
& -\frac{\gamma_{i}(t)}{2} C_{i}(t) .
\end{aligned}
$$


The result, satisfying $C_{i}(-\infty)=0$, then is

$$
\begin{aligned}
C_{i}(t)= & \exp \left[-\frac{c_{2} \sqrt{\pi} w_{0}\left(1+\operatorname{erf}\left[\frac{\sqrt{2} \mathrm{v}_{L} \mathrm{t}}{\mathrm{w}_{0}}\right]\right)}{2 \sqrt{2} v_{L}}\right] \\
& \times \int_{-\infty}^{t} \mathrm{~d} t_{2} c_{1} \exp \left[\frac{c_{2} \sqrt{\pi} w_{0}\left(1+\operatorname{erf}\left[\frac{\sqrt{2} \mathrm{v}_{L} \mathrm{t}_{2}}{\mathrm{w}_{0}}\right]\right)}{2 \sqrt{2} v_{L}}\right] \\
& \times \exp \left[-2 \frac{\left(v_{L} t_{2}\right)^{2}}{w_{0}^{2}}+\mathrm{i} \delta \omega_{c a} t_{2}\right],
\end{aligned}
$$

where the introduced constants $c_{1}$ and $c_{2}$ are:

$$
\begin{aligned}
& c_{1}=\frac{E_{0}^{2}}{2(i \hbar)^{2}} \exp \left[-2 \frac{\rho_{L}^{2}}{w_{0}^{2}}\right] \sum_{b=2,3 \ldots} \frac{\left(\vec{\mu}_{a b} \cdot \vec{\epsilon}\right)\left(\vec{\mu}_{b c} \cdot \vec{\epsilon}\right)}{\mathrm{i}\left(\omega_{b a}-\omega\right)}, \\
& c_{2}=\pi \beta_{\text {ioni }} \epsilon_{0} c E_{0}^{2} \exp \left[-2 \rho_{L}^{2} / w_{0}^{2}\right] .
\end{aligned}
$$

Again, it is useful to express the constants in terms of the laser power, beam waist and fundamental constants, with the absolute values in SI units:

$$
\begin{aligned}
\left|c_{1}\right| & =\frac{2 P}{\pi \epsilon_{0} c \hbar w_{0}^{2}}\left(\frac{11.78}{2} \frac{2 a_{0}^{2} e^{2}}{3 R y}\right) \exp \left[-2 \frac{\rho_{L}^{2}}{w_{0}^{2}}\right] \\
& =2.94 \times 10^{-4} \frac{P}{w_{0}^{2}} \exp \left[-2 \frac{\rho_{L}^{2}}{w_{0}^{2}}\right], \\
\left|c_{2}\right| & =4 \beta_{\text {ioni }} \frac{P}{w_{0}^{2}} \exp \left[-2 \frac{\rho_{L}^{2}}{w_{0}^{2}}\right] \\
& =4.81 \times 10^{-4} \frac{P}{w_{0}^{2}} \exp \left[-2 \frac{\rho_{L}^{2}}{w_{0}^{2}}\right] .
\end{aligned}
$$

An approximate analytical solution to equation (9) can be obtained (by first suppressing the $\left(\mathrm{i} \omega_{c a} t_{2}\right)$ term from the integral and then later incorporating it as if $c_{2}=0$ ) to be:

$$
\begin{aligned}
& C_{i}(\infty) \approx 0.864 \exp \left[-\frac{w_{0}^{2} \delta \omega_{c a}^{2}}{8 v_{L}^{2}}\right] \exp \left[-\frac{c_{2} \sqrt{\pi} w_{0}}{2 v_{L}}\right] \\
& \times\left[\exp \left(4.254 \times 10^{-4} \frac{P}{v_{L} w_{0}} \exp \left[-2 \frac{\rho_{L}^{2}}{w_{0}^{2}}\right]\right)-1\right] .
\end{aligned}
$$

The result expressed by equation (11) is far from rigorous and it should be checked. A comparison of the results from this equation (11) to the numerical integration of equations $(10)(a-c)$ of [35] for a laser power of $5 \mathrm{~W}$ in a beam waist of $100 \mu \mathrm{m}$ and crossing speed of $50 \mathrm{~m} \mathrm{~s}^{-1}$ (and $70 \mathrm{~m} \mathrm{~s}^{-1}$ ) provides the values 0.08947 (and 0.0511 ) against the correct values of 0.0903 (and 0.0519). These values illustrate the validity of equation (11) for the purpose of providing a rough estimate in the scenarios here considered.

Equations (7) and (9) (or equation (11)) can be computed for different trajectories of atoms in the trap through the laser beam with a Monte-Carlo simulation. As an example, we compute the passage of an atom at speeds transverse to the laser beam of $50 \mathrm{~m} \mathrm{~s}^{-1}$ and $70 \mathrm{~m} \mathrm{~s}^{-1}$, with a beam waist of $100 \mu \mathrm{m}$ with varying laser power as shown in figure 1. For lower speeds or for obtaining lineshapes, it is important to incorporate a numerical integrator for the density matrix dynamics in the Monte Carlo simulation.

The fraction of ionized atoms, against excited ones, can be computed by the difference between the curves. A graph against laser power and crossing speed $\left(v_{L}\right)$ is shown in figure 2 using the approximate equations (7) and (11).

A decay rate of the 2 s state induced by electric field, either by the applied field of the Penning trap or by the relativistic field seen by the moving atom in the magnetic trap, can also be roughly incorporated into the equations above by a similar procedure to the inclusion of the photoionization rate. Introducing a decay rate $\gamma_{E}$, which can depend on the electric and magnetic fields and the velocity, equation (9) can be modified to:

$$
\begin{aligned}
C_{i E}(t) \approx & \exp \left[-\frac{\gamma_{E} t}{2}\right] \\
& \times \exp \left[-\frac{c_{2} \sqrt{\pi} w_{0}\left(1+\operatorname{erf}\left[\frac{\sqrt{2} \mathrm{v}_{L} \mathrm{t}}{\mathrm{w}_{0}}\right]\right)}{2 \sqrt{2} v_{L}}\right] \\
& \left.\times \int_{-\infty}^{t}\right] \mathrm{d} t_{2} c_{1} \exp \left[+\frac{\gamma_{E} t}{2}\right] \\
& \times \exp \left[\frac{c_{2} \sqrt{\pi} w_{0}\left(1+\operatorname{erf}\left[\frac{\sqrt{2} \mathrm{v}_{L} \mathrm{t}_{2}}{\mathrm{w}_{0}}\right]\right)}{2 \sqrt{2} v_{L}}\right] \\
& \times \exp \left[-2 \frac{\left(v_{L} t_{2}\right)^{2}}{w_{0}^{2}}+\mathrm{i} \delta \omega_{c a} t_{2}\right] .
\end{aligned}
$$

For the parameters in figure 1 and a decay rate of $\approx 2.8 \times 10^{3} \mathrm{~s}^{-1}$ (corresponding to an applied electric field of $1 \mathrm{~V} \mathrm{~cm}^{-1}$ at $B \approx 0$ ) discussed in section 2 , the change is imperceptible to $\left|C_{i}\right|$ assuming its value right after passing through the beam, since in this case one should not take the infinite time limit where $\left|C_{i E}(\infty)\right| \rightarrow 0$.

\section{References}

[1] Amoretti M et al 2002 Production and detection of cold antihydrogen atoms Nature 419456

[2] Gabrielse G et al 2002 Background-free observation of cold antihydrogen and a field-ionization analysis of its states Phys. Rev. Lett. 89213401 
[3] Enomoto Y et al 2010 Synthesis of cold antihydrogen in a cusp trap Phys. Rev. Lett. 105243401

[4] Andresen G B et al 2010 Trapped antihydrogen Nature 468 673

[5] Andresen G B et al 2011 Confinement of antihydrogen for 1000 seconds Nat. Phys. 7558

[6] Gabrielse G et al 2012 Trapped antihydrogen in its ground state Phys. Rev. Lett. 108113002

[7] Parthey Ch G et al 2011 Improved measurement of the hydrogen 1S-2S transition frequency Phys. Rev. Lett. 107203001

[8] Cesar C L et al 2009 Antihydrogen physics at ALPHA/CERN Can. J. Phys. 87791

[9] Cesar C L et al 1996 Two-photon spectroscopy of trapped atomic hydrogen Phys. Rev. Lett. 77255

[10] Madsen N, Rasmussen C, Robicheaux F et al Aspects of 1s-2s spectroscopy of single trapped antihydrogen atoms, in preparation

[11] Amole C et al 2012 Resonant quantum transitions in trapped antihydrogen atoms Nature 483439

[12] Cesar C L 2011 ALPHA Collaboration Physics Meeting (unpublished)

[13] Cesar C L and Kleppner D 1999 Two-photon Doppler-free spectroscopy of trapped atoms Phys. Rev. A 594564

[14] Cesar C L 2001 Zeeman effect on the 1S-2S transition in trapped hydrogen and antihydrogen Phys. Rev. A 64023418

[15] Bethe H and Salpeter E 1977 Quantum Mechanics of One- and Two-Electron Atoms (Berlin: Springer) (doi:10.1007/9781-4613-4104-8)

[16] Lamb W E Jr and Retherford R C 1950 Fine structure of the hydrogen atom: I. Phys. Rev. 79549

[17] Hermann M, Haas M, Jentschura U D, Kottmann F et al 2009 Feasibility of coherent xuv spectroscopy on the 1S-2S transition in singly ionized helium Phys. Rev. A 79052505

[18] Hess H F, Kochanski G, Doyle J M, Masuhara N, Kleppner D and Greytak T J 1987 Phys. Rev. Lett. 59672

[19] Setija I D et al 1993 Optical cooling of atomic hydrogen in a magnetic trap Phys. Rev. Lett. 702257

[20] Cesar C L, Fried D G, Killiam T C, Polcyn A D, Sandberg J C, Yu I A, Greytak T J, Kleppner D and Doyle J M 1996 Twophoton spectroscopy of trapped atomic hydrogen Phys. Rev. Lett. 77255

[21] Hancox C I, Doret S C, Hummon M T, Luo L and Doyle J M 2004 Magnetic trapping of rare-Earth atoms at millikelvin temperatures Nature 431281
[22] Hogan S D, Wiederkehr A W, Schmutz H and Merkt F 2008 Magnetic trapping of hydrogen after multistage Zeeman deceleration Phys. Rev. Lett. 101143001

[23] Wiederkehr A W, Hogan S D, Lambillotte B, Andrist M, Schmutz H, Agner J, Salathe Y and Merkt F 2010 Trapping deuterium atoms Phys. Rev. A 81021402

[24] Lambo R, Rodegheri C C, Silveira D M and Cesar C L 2007 Spectroscopy of low-energy atoms released from a solid noble-gas matrix: proposal for a trap-loading technique Phys. Rev. A 76061401

[25] Crivelli P, Cesar C L and Lambo R 2009 A new trap loading mechanism for hydrogen Can. J. Phys. 87 799-806

[26] Sacramento R L, Scudeller L A, Lambo R, Crivelli P and Cesar C L 2011 Spectroscopy of lithium atoms sublimated from isolation matrix of solid Ne J. Chem. Phys. 135 134201

[27] Sacramento R L, Alves B X, Almeida D T, Wolff W, Li M S and Cesar C L 2012 Source of slow lithium atoms from $\mathrm{Ne}$ or $\mathrm{H} 2$ matrix isolation sublimation J. Chem. Phys. 136154202

[28] Oliveira A N, Sacramento R L, Alves B X, Silva B A, Wolff W and Cesar C L 2014 Slow ground state molecules from matrix isolation sublimation J. Phys. B: At. Mol. Phys. 47245302

[29] Sacramento R L, Oliveira A N, Alves B X, Silva B A, Li M S, Wolff W and Cesar C L 2015 Matrix isolation sublimation: An apparatus for producing cryogenic beams of atoms and molecules Rev. Sci. Instrum. 86073109

[30] Bovino S, Zhang P, Kharchenko V and Dalgarno A 2009 Trapping hydrogen atoms from a neon gas matrix: a theoretical simulation J. Chem. Phys. 131054302

[31] Cesar C L, Sacramento R L and Silveira D M 2013 Proposal for $\mathrm{H}$ trapping at ALPHA-II during pbar shutdown period and $\mathrm{H}$ reference in ALPHA Collaboration Physics Meeting (unpublished)

[32] Plimmer M D 2004 Optical frequency standards based on molecules, atoms and ions Phys. Scr. $70 \mathrm{C} 10$

[33] Yariv A 1989 Quantum Electronics (New York: Wiley)

[34] Bassani F, Forney J J and Quattropani A 1977 Choice of gauge in two-photon transitions: $1 \mathrm{~s}-2 \mathrm{~s}$ transition in atomic hydrogen Phys. Rev. Lett. 391070

[35] Haas M et al 2006 Two-photon excitation dynamics in bound two-body Coulomb systems including ac Stark shift and ionization Phys. Rev. A 73052501 